logos_i_ethos_2_(33)_2012, s. 149-177

Krzysztof Skorulski

\title{
Am „Leitseil des Wortes" zum Verständnis des Menschen. Denken mit Ferdinand Ebner und Józef Tischner ${ }^{1}$
}

Die Frage nach dem Menschen ist angesichts der heutigen Veränderungen in seinem Verständnis immer aktuell. Grundsätzlich wird mit dem Menschen entweder auf eine reduktionistische Weise umgegangen, indem man ihn nämlich „von außen”, von der Seite seiner Organe, Funktionen, Verhalten usw. betrachtet, oder man versucht ihn "ganzheitlich", „von innen” zu sehen, nach dem, was "den Menschen ausmacht” zu

Krzysztof Skorulski (1962) - geboren in Polen, lebt in Innsbruck. Promoviert in Theologie an der Universität Innsbruck, Aktivitäten in der Internationalen Ferdinand Ebner Gesellschaft, wo er gegenwärtig der Vorstand der Forschungssektion ist. Zahlreiche Übersetzungen ins Polnische u.a. der Werke von Henri Bergson und Wort und die geistigen Realitäten von Ferdinand Ebner. Einige Veröffentlichungen, vor allem in Polen im Bereich Philosophie und Spiritualität (Schwerpunkt Ferdinand Ebner).

suchen. Die Ansätze sowohl Ferdinand Ebners, des „Begründers der Dialogphilosophie”, als auch Józef Tischners, des polnischen „Denkens der Solidarität" können eindeutig innerhalb der zweiten Sichtweise lokalisiert werden.

Was haben aber die beiden Denker gemeinsam? Eine provisorische Antwort ist überraschend einfach: das dialogische Konzept des Menschen. Dennoch kann man die Unterschiede zwischen ihnen nicht übersehen. Tischner sieht die Situation des Menschen wesentlich als

Dieser Text ist die ausgeweitete und für den deutschsprachigen Leser angepasste Version meines Referats, welches während des Tischner-Kongresses in Kraków am 25-27.10.2011 auf Polnisch gehalten wurde.

2 Vgl. J. Tischner, Ethik der Solidarität. Prinzipien einer Hoffnung, Graz 1982. 
Das menschliche Drama ${ }^{3}$, das sich auf der Bühne der Welt (die Welt der Objekte) zwischen Personen abspielt. In diesem Drama geht es für den Menschen um etwas: in der ersten Linie um das Gute, das nur in der Freiheit möglich ist. Ein guter Abschluss des Dramas kann gelingen, muss aber nicht. Daher ist auch die Hoffnung berechtigt, dass sich dieses Drama gut ausgeht. Tischner wird eine besondere Fähigkeit zugeschrieben, auf die wesentlichsten Worte seiner Zeit einzugehen und sie zu seinem Thema zu machen. Ebner dagegen scheint sich in seinem Denken mit der ganzen Konsequenz „spiralförmig” um sein „dialogisches Prinzip”, wie ein Planet um die Sonne zu drehen, wobei er immer neue Gebiete einbezieht. Um sich jetzt die Stärken beider Autoren zunutze zu machen, wird in diesem Text versucht, drei der Hauptthemen Tischners: das Gute, die Freiheit, die Hoffnung (man könnte sicher auch andere hinzufügen, z. B. die Wahrheit) mit der Ebner'schen Denkweise („Denken am Seil der Sprache/des Wortes") zu reinterpretieren. Somit soll eine vertiefte dialogische Sicht des Menschen entworfen werden.

\section{1. „Denken am Leitseil des Wortes”}

Ebner wird in seinen Überlegungen von dem Motto „Denken am Leitseil der Sprache"4 begleitet. Diesen Satz kann man entweder als eine Feststellung, oder als einen Wunsch interpretieren. Wenn wir ihn als Feststellung nehmen ${ }^{5}$, haben wir es mit einer Aussage im Stil der "Sprachkritik"

3 Vgl. Ders., Das menschliche Drama: Phänomenologische Studien zur Philosophie des Dramas, übers. von S. Dzida, München 1989, S. $21 \mathrm{ff}$.

4 „Denken am Leitseil der Sprache - Sprache als Depot sprachgewordener Gedanken” (30.07.1921). F. Ebner, Notizen, [in:] Ders., Schriften, Bd. 1, München 1963, S. 459.

5 So scheint z. B. Karl Wucherer zu suggerieren. Vgl. A. K. Wucherer-Huldenfeld, Personales Sein und Wort, Graz-Wien 1985, S. 39.

$6 \quad$ Ich meine hier die Geistesströmung, unter deren Repräsentanten Denker wie Ernst Mach, Fritz Mauthner, Karl Kraus oder Ludwig Wittgenstein genannt werden können. Vgl. K. Skorulski, Wort contra Begriff: Der schwindende Sinn der Worte und die Anfänge der Dialogphilosophie, [in:] Pneumatologie als Grammatik der Subjektivität: Ferdinand Ebner, Wien 2012, S. 51ff. Laut der „Sprachkritik” können wir zwischen dem Denken und der Sprache die Gleichheit feststellen, wie es z. B. Mauthner in seinen „Beiträgen zu einer Kritik der Sprache” wiederholt. „Da Denken nichts 
zu tun, die die Abhängigkeit des Denkens von der Sprache behauptet. In unserem Fall ist es aber förderlicher, in diesem Satz einen Wunsch zu sehen und uns auf das Bild einer Wanderung im Hochgebirge zu berufen, wo der Kletterer für jeden Stützpunkt dankbar sein muss und versucht, sich an dem Sicherheitsseil zu halten. Dieses Bild gibt meines Erachtens besser die Situation Ebners wieder, der in der Zeit eines Umbruchs der Kultur und der Zivilisation lebte - welche von einem massiven Sinnverlust der Worte, von einer Krise der Sprache gekennzeichnet war - und durch das Halten am Wort einen Sturz ins abstrakte Denken, in den Abgrund der leeren Worte vermeiden will?

Tatsache ist nämlich, dass der Ebner'sche Versuch, den Sinn der Worte zu retten - im Unterschied zu den in seiner Zeit populären Ideen der "Sprachkritik”, welche die Sprache als eine Art ideelle Struktur sehen - sich schon in seinem Ausgangspunkt nicht für die Sprache als ein System der Begriffe, sondern für die im Alltag gesprochene, lebendige Sprache interessiert. Ebner fragt, was das Wesen des Wortes - als Einheit der Sprache - im geistigen Sinne ${ }^{8}$ ausmacht, und was das Wort in der menschlichen Welt bewirkt. Auch seine Antworten auf diese Fragen weisen eine ganz andere Richtung als die der Sprachkritik. Fundamental ist dabei die Unterscheidung zwischen dem Wort im „technischen Sinne” (Wort, im Plural „Wörter”) und dem „rechten Wort” (Wort, im Plural „Worte"). Das erstere war Objekt der sprachwissenschaftlichen Analysen und der "Sprachkritiker”. Diese Wörter werden leicht zu „leeren Wörtern”, „abstrakten Begriffen”, die vielleicht miteinander in einem logischen $\mathrm{Zu}-$ sammenhang stehen, aber keinen Bezug auf die konkrete Situation des Lebens haben. Ebner interessiert sich vor allem für das Wort im zweiten Sinne: dieses Wort ist ein in einer konkreten Lebenssituation gesprochenes Wort, welches sich als wirksam erweist. Wenn ich an jemanden ein

anderes ist als Sprechen.... F. Mauthner, Beiträge zu einer Kritik der Sprache, Bd. 2: Zur Sprachwissenschaft, Stuttgart 1912, S. 21. Vgl. ebenda, S. 661f.

Vgl. K. Skorulski, Wort contra..., op. cit., S. $49 f$.

Das Ebner'sche Verständnis „des Geistigen” im Menschen unterscheidet sich jedoch - wie wir sehen werden - vom typischen Verständnis des Geistes in der deutschsprachigen Philosophie und konzentriert sich auf dem dialogischen, zwischenmenschlichen Aspekt. 
solches Wort richte, trete ich mit ihm in Beziehung. Dieses Wort kann man also auch Satz, Sprache, Beziehung nennen, und der Ursatz ist laut Ebner „Ich bin”. Da also das „Wort” in diesem Sinne oft gleichbedeutend mit „Sprache” ist, ist es auch kein Fehler, die „Sprache” durch „Wort” zu ersetzen und von einem „Denken am Leitseil des Wortes” zu sprechen.

Was bedeutet also das „Denken am Leitseil des Wortes”? Versuchen wir das Eigene des Ebner'schen Denkens als eines „an das Wort als Sicherheitsseil sich klammernden” zu fassen. Das „Denken am Leitseil des Wortes” muss im Licht des Ebner'schen „Grundgedankens” interpretiert werden, so wie er am Anfang seines Hauptwerks steht:

Vorausgesetzt, daß die menschliche Existenz in ihrem Kern überhaupt eine geistige, [ist] (...) so ist dieses wesentlich dadurch bestimmt, daß es vom Grund aus angelegt ist auf ein Verhältnis zu etwas Geistigem außer ihm, durch das es und in dem es existiert. Ein Ausdruck, und zwar eben der „objektiv” faßbare und darum einer objektiven Erkenntnis zugängliche Ausdruck des Angelegtseins auf eine derartige Beziehung ist in der Tatsache zu finden, daß der Mensch ein sprechendes Wesen ist, daß er das „Wort hat.” (...) Wenn wir nun, um ein Wort dafür zu haben, dieses Geistige im Menschen Ich nennen, das außer ihm aber, zu dem im Verhältnis das „Ich” existiert, Du, so haben wir zu bedenken, daß dieses Ich und dieses Du uns eben durch das Wort und in ihm in seiner „Innerlichkeit” gegeben sind; nicht jedoch als „leere” Wörter, denen kein Bezughaben auf eine Realität innewohnte - als was sie freilich in ihrem abstrakten, substantivierten und substantialisierten Gebrauche bereits erscheinen-, vielmehr als Wort, das in der Konkretheit und Aktualität seines Ausgesprochenwerdens in der durch das Sprechen geschaffenen Situation seinen „Inhalt” und Realitätsgehalt „redupliziert”. Das ist in Kürze der Grundgedanke9.

Dieser „Grundgedanke” ist ein Versuch, das Ebner'sche Konzept in eine möglichst kurze Formel $\mathrm{zu}$ fassen und als solches wird es uns in unseren Ausführungen begleiten.

9 F. Ebner, Das Wort und die geistigen Realitäten: Pneumatologische Fragmente, Wien 2009, S. 14. 


\subsection{Dialogizität des Wortes}

Die Aussage Ebners bedeutet erstens zweifellos nichts anderes als dass es kein „reales” Ich „ohne Du” gibt - alle Arten des einsamen „absoluten Ich", wie wir sie aus dem deutschen Idealismus kennen, sind nicht mehr als intellektuelle Konstrukte, „leere Wörter”. Zweitens aber, dieses Ich und $\mathrm{Du}$ - Ebner nennt sie die „geistigen Realitäten” im Unterschied zu den Wortfiktionen, suggeriert also, dass ihnen in der realen Welt etwas entspricht - sind uns, und zwar „objektiv”, durch Wort und im Wort gegeben, im Wort „in der Konkretheit und Aktualität seines Ausgesprochenwerdens”, also „in der durch das Sprechen geschaffenen Situation”. Das Wort also, wenn es sich an der Wirklichkeit halten will, muss immer die Gegenwart sowohl des Ich als auch des $\mathrm{Du}$ „in der durch das Sprechen geschaffenen Situation" voraussetzen. Um es kurz zu fassen: wenn das Ich als untrennbar vom Du erscheint, dann wenn ich „Ich” denke, muss ich gleichzeitig „Du” denken, genauer: „das Ich im Verhältnis zum Du". In der Aktualität der Sprache sehen wir, dass sogar ein Ich in der „Icheinsamkeit” - wie Ebner es nennt - „hinter seiner chinesischen Mauer eingeschlossen", immer mit einem gewissen $\mathrm{Du}$ zu tun hat, ob das ein „pathologisches”, ideelles oder fiktives Du sein mag. Ein „Du” ist immer vorhanden, auch wenn es nur eine Projektion des „Ich" ist.

Erst nach dem ich gemerkt habe, dass meinem Ich immer ein Du entspricht, kann ich tatsächlich mein reales Ich bemerken, ohne es zum abstrakten Subjekt zu reduzieren. Und im Gegenteil, wenn ich mein reales Du nicht sehe, oder wenn ich es zum Verschwinden bringen will, zur „Objektivierung", verwische ich auf eine gewisse Weise auch mein reales Ich und bewege mich im Bereich der „leeren Wörter”. Es sind zwei wesentlich unterschiedliche Situationen, einerseits wenn wir streiten, wie wir das Wort „Hoffnung” definieren sollen, und andererseits wenn ich ein hoffender Mensch bin, oder genauer "Ich hoffe" (mit verbum finitum) sage - zu einem realen, konkreten, gegenwärtigen Du. Die zweite Situation erscheint als die ursprüngliche. Wenn ich voller Hoffnung bin, denke ich nicht an den Hoffnungsbegriff, sondern lebe oder erlebe sie, und der Ausdruck dieser Situation ist die an ein Du gerichtete Aussage 
„Ich hoffe”. Hier kann ein Denken ansetzen, das nicht von der Realität der menschlichen Existenz getrennt sein will.

„Denken am Leitseil des Wortes” bedeutet also die „Dialogizität”, das Berücksichtigen des $\mathrm{Du}$, im Unterschied zu dem „Monologismus" - einem Versuch, das Du zu verwischen. Jeder wortwerdende Gedanke geschieht nicht in einem einsamen Ich, sondern richtet sich an ein $\mathrm{Du}$ - auch wenn es uns nicht immer bewusst ist. Es gibt keinen ganz einsamen Gedanken - vielleicht mit Ausnahme der mathematischen Formel.

\subsection{Aktualität des Wortes}

Ebner meint das Wort „in der Konkretheit und Aktualität seines Ausgesprochenwerdens", dieses dialogische Wort hat also seine zeitliche (nicht „außerzeitliche”, wie die Idee) Dimension. Bereits der „Ursatz” „Ich bin” offenbart seinen richtigen Sinn erst dann, wenn er in der Aktualität seines Ausgesprochenwerdens verstanden wird. Versuchen wir, diesen Ausdruck zu erläutern. Es ist kein Geheimnis, dass einzelne dialogische Aussagen unterschiedliche Aktualitätsgrade aufweisen, abhängig von solchen Eigenschaften wie Wichtigkeit, Wahrheit, Ernst, Verständlichkeit, zeitliche und räumliche Nähe, und gleichzeitig von solchen Eigenschaften des „Angesprochenen” wie Offenheit und Respekt gegenüber dem Sprechenden, sowie Bereitschaft, ihm zu glauben. Je größer die Wichtigkeit, Wahrheit usw. der Aussage, desto größer ihre Aktualität ${ }^{10}$. Die Aktualität des Wortes, von der Ebner spricht, setzt den höchsten Grad der genannten Eigenschaften voraus ${ }^{11}$. Diese Aktualität ist - um es bildlich zu zeigen - das Gegenteil der Aussage eines Papageis, der „Ich bin Professor” sagt. Eine solche

10 Wucherer zeigt in seiner Analyse am Beispiel des Rufes „Es brennt!” ein ganzes Spektrum solcher Situationen, angefangen von dem Kinderspiel „warm-kalt” bis zum wirklichen Brand, wo sowohl der Sprechende als auch der Hörende vor einem brennenden Haus stehen. Vgl. K. Wucherer-Huldenfeld, Personales Sein..., op. cit., S. $45 f$.

11 „Vom Bezug des Wortes zum Geschehen im höchsten Grade des Ernstes ist auszugehen, wenn wir das Leben des Wortes im Sinne Ebners verstehen wollen” (ebenda, S. 46). 
Aussage - durch den Mangel an Aktualität - bedeutet nicht, was sie aussagt.

Um zusammenzufassen sagen wir noch einmal, dass das „Denken am Leitseil des Wortes" diese Aktualität des Wortes berücksichtigen, d. h. das Wort als aktuell, gesprochen, lebendig, in einer konkreten, aktuellen Beziehung zum Du sehen muss.

\section{3 „Göttlichkeit” des Wortes}

„Denken am Leitseil des Wortes” bedeutet auch die Offenheit der Beziehung des Ich zum Du auf eine breitere Dimension hin anzuerkennen, im Gegensatz zu einer dicht abgeschlossenen „Dyade” (analog zur "Monade" bei Leibniz und Tischner). Inspiriert durch den Johanneischen Logos fragt Ebner nach dem Anfang der Sprache, Anfang des Wortes. Der Mensch merkt, dass er immer schon angesprochen ist; bevor er zum Ich wird, ist er bereits das Du eines „von außen kommenden” Ich. Auf diese Weise öffnet sich das Ebner'sche Konzept auf ein „absolutes” Ich im „metaphysischen” Sinne (hier anders als bei Ebner, der unter „Metaphysik" eine Spekulation mit Wörtern versteht), und genauer auf das „erste” Ich, das den Menschen anspricht, oder sogar „schafft, indem es zu ihm spricht" - also auf Gott ${ }^{12}$. Nach der biblischen Logik müssen wir hinzufügen, dass kein anderer als Jesus Christus das Wort Gottes an den Menschen ist. Wenn ich im Wort den Anderen „objektiv” bemerke, dann ist mir auch im Wort (Jesus Christus) Gott gegeben. Eine Begegnung mit dem anderen Menschen ist zugleich eine Begegnung mit Gott. Diese Vision der Beziehung des Menschen mit Gott ist auch für Theologen inspirierend gewesen, wenn wir nur solche wie Karl Rahner ${ }^{13}$ oder Hans Urs von Balthasar ${ }^{14}$ nennen. Kurz gesagt, „Denken am Leitseil des Wortes” bedeutet Offenheit hin auf die göttliche Dimension.

12 Vgl. F. Ebner, Das Wort..., op. cit., S. 21.

13 Vgl. K. Rahner, Hörer des Wortes. Zur Grundlegung einer Religionsphilosophie, München 1941, S. 68f, $86 f$.

14 Vgl. S. Zucal, Ferdinand Ebner. La „nostalgia” della parola, Brescia 1999, S. $206 f$. 


\subsection{Wahrheit des Wortes}

Wenn wir am Leitseil des Wortes denken, meinen wir das wahre Wort. Ebner gibt sich nicht mit der überkommenen Wahrheitsdefinition zufrieden, sondern sucht nach einer solchen Sicht, die am ursprünglichsten für das „Zwischenmenschliche” ist. Manchmal formuliert er sie als adequatio, und zwar adequatio der Aussage und der Persönlichkeit, der Aussage und des Seins. Die Aussage muss von der Persönlichkeit "gedeckt sein”, d. h. ich muss, aussagend, mit meinem Leben das Ausgesagte „decken”. Gleichzeitig habe ich aber die Möglichkeit, es nicht zu „decken”. Was passiert dann? Ich verschließe mich vor dem, zu dem ich spreche. Ich bin, so zu sagen, allein mit meiner Wahrheit, und nach Außen zeige sich etwas Anderes - aber gerade dadurch „bin ich einsam”. Kurz gesagt - „ich lüge”. Mein Verhältnis zum Du ist verfälscht, „,ist also de facto nicht”, ich bleibe in der „Icheinsamkeit”. Ich bin für mein Du in meinem Wort nicht „objektiv gegeben”.

Eine andere Weise, die Bedeutung der Wahrheit zu verstehen, führt durch die Beschreibung des Weges vom Gedanken zum Wort. Zuerst wird in mir der Gedanke geboren. Wir lassen hier die Frage beiseite, ob wir immer schon in Worten denken, sowie eine allgemeinere Frage, ob unser Denken durch unsere Sprache bedingt ist oder umgekehrt ${ }^{15}$. Wenn wir auch nicht immer in Worten denken, dann jedenfalls je näher zum Wort, desto klarer, konkreter unser Gedanke, und wenn er Wort wird, kann er auch wahr sein. Jedoch

Alles, was im Menschen, je mehr es aus der Tiefe des Lebens kommt, Wort wird, hat seinen rechten Sinn und seine eigentliche Wahrheit darin, dass es das Du nicht verfehlt. (...) es gibt keine Wahrheit eines wortgewordenen Gedankens, die absolut unabhängig von der Beziehung des Wortes zum Du bestünde,

15 Ich meine hier die Diskussion zwischen dem Standpunkt, dessen Exponent z. B. Noam Chomsky ist (die „universale Grammatik”) und dem Standpunkt z. B. Nietzsches („unser Glaube hängt von der Grammatik ab"), dessen Exponent in der Sprachwissenschaft z. B. Emile Benveniste ist. Erinnern wir auch daran, dass bereits in den Zeiten Ebners für die Sprachkritik Denken=Sprache. 
das es, ideell oder konkret, anspricht. (...) Die Beziehung zum rechten Du ist es, die den wortgewordenen Gedanken zur „objektiven” Wahrheit macht ${ }^{16}$.

Die Wahrheit ist also persönlich, sie ist eine „Wahrheit des Lebens” und als solche ist sie nur in dem Verhältnis des Ich zum Du möglich. Sie besteht in dem Offenbaren meiner Gedanken, also meiner selbst (meines Ich) - dem Anderen im Wort. Hier wird sie also als eine eigene aletheia verstanden, als mein „sich zeigen” in dem Verhältnis zum Du, „sich zeigen” im Wort. Ebner schreibt:

In der Icheinsamkeit seiner Existenz kann der Mensch nicht die Wahrheit seines Lebens haben. Denn diese existiert nur im Medium des „Wortes”, im ursprünglichen und wesentlichen Angelegtsein des Geistigen im Menschen zu etwas Geistigem außer ihm, im Verhältnis des Ichs zum $\mathrm{Du}^{17}$.

Zusammenfassend kann man also sagen, dass die Wahrheit für Ebner ein „Gegeben-sein”, „sich Offenbaren”, eine Offenheit des realen Ich bedeutet - selbstverständlich im Wort. Man kann sogar noch weiter gehen und allgemeiner sagen: Die Wahrheit ist das „Offenbarwerden alles Seins im Wort"18.

\subsection{Wort und Liebe}

Ein Denken am Leitseil des Wortes muss desweiteren berücksichtigen, dass das Wort im Menschen auf eine grundlegende Weise mit Liebe verbunden ist. Das Wort ist kein wahres Wort ohne Liebe. In seinem Hauptwerk schreibt Ebner:

So gehören denn das Wort und die Liebe zusammen. Zwar schöpft der Mensch aus jenem und der durch es in ihn gelegten Vernunft alle Kraft des Er-

16 F. Ebner, Das Wort..., op. cit., S. 42.

17 Ders., Schriften, Bd. 2, op. cit., S. 186.

18 K. Wucherer-Huldenfeld, Personales Sein..., op. cit., S. 262. 
kennens, im letzten Grunde jedoch seines Ihm-Gegebenseins dient es der Liebe, der göttlichen Liebe und der von Gott geforderten Nächstenliebe. Das rechte Wort ist immer eines, das die Liebe spricht, und es wohnt ihm die Kraft inne, chinesische Mauern zu durchbrechen. (...) Das lieblose Wort aber ist bereits menschlicher Mißbrauch mit der göttlichen Gabe des Wortes. In ihm streitet das Wort wider seinen eigenen Sinn und hebt geistig sich selbst auf ${ }^{19}$.

Die Realitäten des geistigen Lebens, d. h. Ich und Du haben ihr objektives Sein im Wort, und subjektives - in der Liebe. „Dessen Objektiv-im-Wort-Gegebensein entspricht sein «subjektiver» Bestand in der Liebe; so daß also das Wort und die Liebe in ihrem geistigen Grunde zusammengehören"20. Um die Aufgabe der Herstellung des Verhältnisses des Ich zum Du zu erfüllen genügt also nicht ein „beliebiges” Wort: es geht um das mit Liebe verbundene Wort, um das „rechte Wort”. Ohne Liebe verbindet das Wort nicht mit dem Anderen als Du, und kann sogar töten.

Wenn wir das Wort genau in dem von Ebner gebrauchten Sinne verstehen, gehört die Liebe bereits dazu. Die Liebe wird hier mit Ebner - vielleicht minimalistisch - verstanden als eine Offenheit vor dem (realen) Anderen, eine positive Einstellung ihm gegenüber, als ein Respekt ihm gegenüber. Diese positive Einstellung ist nur subjektiv „von Innen” feststellbar, von außen ist sie lediglich in Worten und im Verhalten der betreffenden Person uns zugänglich, die ja auch „verfälscht” werden können. Ohne Liebe ist das Wort kein „rechtes Wort”21 und kann das Du als eine geistige Realität nicht „treffen”, hat dadurch keine wirkende Kraft und baut kein Verhältnis zum realen Du auf. Das Wort und die Liebe sollen als beide Seiten der gleichen Medaille gesehen werden. Wenn wir also vom „rechten Wort” reden, meinen wir auch die in ihm wohnende Liebe. Obwohl man sich durchaus ein Wort ohne Liebe vorstellen kann (Buber schreibt z. B. von dem „technischen Dialog” - Dialog ohne Liebe ${ }^{22}$ ), ist

19 F. Ebner, Das Wort..., op. cit., S. $92 \mathrm{f}$.

20 Ebenda, S. 25.

21 Ebenda, S. 93.

22 Vgl. M. Buber, Zwiesprache, [in:] Ders., Die Schriften über das Dialogische Prinzip, Heidelberg 1954, S. 152. 
ein solches Wort die Verneinung des Sinnes des Wortes, denn es baut kein Verhältnis des Ich zum Du auf. Und wenn auch eine Liebe ohne Worte vorstellbar ist, kann es keine wahre Liebe sein, vielmehr eine Phantasie des Ich. In diesem Sinne kritisiert Ebner Goethe wegen Philines Aussage aus „Wilhelm Meisters Lehrjahren”: „Wenn ich dich liebe, was geht's dich an?"23.

Die Tatsache, dass Ebner auf das (so oft missbrauchte) Wort „Liebe” in seiner Erhabenheit aber auch Vieldeutigkeit zugreift, hat offensichtlich konkrete Gründe. Auf diese Weise hat er eine Möglichkeit, die grundlegende Einheit des Menschen zu betonen. Auf Liebe aufbauend, kann er an die Ursehnsucht des Menschen appellieren, die sowohl in der Psychologie, Metaphysik oder Religion anwesend ist, den Menschen „positiv”, als „das Gute” zu sehen.

\subsection{Wort und Person - das Konzept des Menschen}

Das Ebner'sche Konzept des Wortes ist gleichbedeutend mit dem Konzept des Menschen. Die These der Ebner'schen Anthropologie lautet: Der Mensch hat das Wort. Das Wort bedeutet aber die Möglichkeit, ein Du anzusprechen und von einem Du angesprochen zu werden, d. h. das Du eines Ich zu werden. Ein wahres, nicht nur vorgetäuschtes „Du” kann man nur in der Liebe sein. Auf diese Weise bedeutet „der Mensch hat das Wort” auch: er ,,ist angelegt auf ein Verhältnis zu etwas Geistigem außer ihm”. Das Wort zu haben heißt gleichzeitig „Person zu sein”. Dies wird besonders beim Unterschied zwischen der Aussage in der ersten und zweiten und der Aussage in der dritten Person der Grammatik sichtbar:

Diese gedanklich nicht aufzuhebende Identität des Subjekts der Aussage und des Prädikats macht das Wesen der Existentialbehauptung in der ersten und zweiten Person aus, in der eben ein Sein im Sinne der Personalität behauptet wird, das an und für sich die Beziehung zur Aussage, zum Wort in sich begreift, weil Persönlichkeit und „Wort haben” ein- und dasselbe sind ${ }^{24}$.

23 F. Ebner, Wort und Liebe, [in:] Ders., Schriften, Bd. 1, op. cit., S. 926.

24 Ders., Das Wort..., op. cit., S. 138. 
Zusammenfassend sagen wir, dass eine Person zu sein heißt, zu einem konkreten Du „Ich bin” oder „Du bist” sagen zu können, und zwar aktuell, wahr, liebevoll, so dass diese Aussage sinnvoll und lebendig ist.

\section{Das Gute, Freiheit und Hoffnung vom Blickpunkt des Ebner'schen Wortes}

Die Ebner'sche Suche nach dem richtigen Konzept des Menschen führt ihn zum „rechten Wort”, zum Wort mit Liebe. Tischner entwickelt kein Konzept des Wortes, spricht aber von dem Guten, der Freiheit und der Hoffnung. Können wir aber nicht am Fundament des Guten, der Freiheit und der Hoffnung das „rechte Wort” im Ebner'schen Sinne entdecken?

Wir werden jetzt also die drei großen Themen mit dem dahinter stehenden Menschenbild betrachten, so wie sie der Krakauer Philosoph konzipiert hat, aber mit der Verwendung des Ebner'schen Wortes. Die Reihenfolge unserer Darstellung wird allerdings umgekehrt zu der historischen Entwicklung seines Denkens sein. Am Anfang stellen wir nämlich das Gute (in einem gewissen Sinne der Abschluss Tischners Philosophie), als den Schlüssel zum ganzen menschlichen Drama dar, also auch zur Freiheit und Hoffnung. Nachher betrachten wir die Freiheit, die auch für das Verständnis der Hoffnung nützlich sein wird. Schließlich schreibt Tischner: „Wo Freiheit ist, da gibt es auch Hoffnung" 25 .

\subsection{Das Gute}

Wenn Tischner über das Gute schreibt, dann tut er es angesichts der Unfähigkeit der Ontologie, eine adäquate Beschreibung, ein ausreichendes Verständnis des Menschen zu liefern. Bei solchen typisch menschlichen Themen wie z. B. Verantwortung oder Gnade können so einerseits wichtige Aspekte „der Analyse entgehen, andererseits könnten

25 J. Tischer, Der Streit um die Existenz des Menschen. Aus dem Polnischen und mit einer Einfühung von Steffen Huber, Berlin 2010, S. 348. 
Probleme hervortreten, die uns von der Ontologie aufgezwungen worden sind"26. Er versucht - in Anlehnung an die alten Transzendentalien und an Levinas - ,jenseits von Sein und Nichtsein"27 eine Antwort zu suchen, und er kommt auf alle Fälle einen Schritt weiter, wenn er den Menschen im Licht des Guten sieht. Daher betreibt er neben der Ontologie eine „Agathologie”, die Lehre von dem Guten.

Ebner dagegen bewegt sich in seinem Versuch, den Menschen zu erfassen, „außerhalb aller Transzendentalien”, auch „außerhalb des Guten”: „Nicht die Schönheit und nicht die Idee, auch nicht die Wahrheit - die sollen wir erkennen - und das Gute - das sollen wir tun; nicht die Natur und die Kunst, nicht die Weisheit und nicht die Wissenschaft ist das Objekt der Liebe, sondern das Du"28. Dennoch können wir, glaube ich, ohne größere Schwierigkeiten annehmen, dass sich Ebner zum Betrachten des $\mathrm{Du}$, des anderen Menschen, des biblischen „Nächsten” (auch) als eines "Guten” überreden ließe und zwar in einem einfachen Sinne: Wenn ich nämlich einem $\mathrm{Du}$ antworte oder ein $\mathrm{Du}$ anspreche, erwähle ich es gewissermaßen. Wenn ich es wähle, sehe ich an ihm (auch) Gutes. „Du” ist dabei ein außergewöhnliches „Gutes”. Vom Anfang an drängt es sich uns auf, das Du als ein sich selbst begründendes Gutes zu sehen. In diesem Kontext könnte man sogar von einem „absoluten Guten” sprechen (indem wir darunter einfach ,etwas Autonomes, Selbstverständliches, von Kontexten und Bezügen Unabhängiges" ${ }^{\prime 29}$ verstehen, und vorläufig die Frage nach Gott als dem absoluten Guten beiseite lassen). In diesem Sinne erscheint mir der Mensch als Gutes, auch wenn ihn nichts anderes (höheres?) begründet. Diese Sicht der Dinge gründet im Erleben des Anderen selbst, das analog zum „kategorischen Imperativ” eines Kant oder zum „Antlitz” eines Levinas wirkt.

Man kann natürlich mit Levinas (und Tischner) von einem Anderen, der in „meinem Inneren anwesend ist” und „vom Anfang an dort

26 Ebenda, S. 153.

27 Ebenda, S. 316.

28 F. Ebner, Das Wort..., op. cit., S. 144.

29 B. Korzeniewski, Absolut jako odniesienie urojone, [in:] http://www.racjonalista.pl/ kk.php/s,3724/q,Absolut.jako.odniesienie.urojone, übers. von K. Skorulski (1.07.2012). 
wohnt" sprechen ${ }^{30}$, wenn ich aber einen realen Anderen, ein reales Du meine, und nicht nur seinen Begriff, dann muss ich fragen, wie ist er $\mathrm{zu}$ meinem Inneren gelangt, wenn nicht durch Wort? Wenn ich also, das Wort verschmähend, behaupte, dass der Andere immer schon in mir war, meine ich entweder den Begriff des Anderen, oder berufe ich mich auf das religiöse Denken, auf Gott, auf das göttliche Gesetz. Im ersten Fall habe ich immer noch mit dem Selbem (le même) und nicht mit dem Anderen (lautre) zu tun, also - um mit Ebner zu sprechen - denke ich immer noch in der Icheinsamkeit, auch wenn ich die Möglichkeit einer Offenheit gegenüber dem Du zulasse. Im zweiten Fall begeben wir uns auf das Gebiet der Theologie.

Kurz gesagt: die Tatsache, dass Du, dass der Andere mir als das Gute gegeben ist, wird mir durch das Wort ermöglicht, er ist mir im Wort gegeben. Wenn der Andere in mir ist, dann deswegen, weil ich als Mensch „das Wort habe” und weil er mich in seinem Wort erreicht. Wir könnten also die Aussage von dem Anderen als einem „sich selbst begründenden” Guten präzisieren: ich erlebe das Du (den Anderen) als das (besondere) Gute, weil - wie Ebner schreibt - „er das Wort hat” und „ich das Wort habe”. Er hat das Wort - deshalb ist er für mich das Gute, ich habe das Wort - deshalb kann ich ihn als das Gute sehen. Das Wort hätte also eine besondere Funktion: ohne Wort wäre mir ein Du als das Gute nicht zugänglich, also - weil wir vom Guten in der menschlichen Welt reden - wäre mir gar kein Gutes zugänglich.

Bezieht sich also alles Gute zum Wort in seiner Dialogizität, d. h. zur Welt der zwischenmenschlichen Beziehung, im Unterschied zum einsamen Ich? Jemand könnte ja sagen, dass es andere Möglichkeiten des Guten gibt (z. B. die sog. „Güter dieser Welt”), die „mein Gutes” werden, wenn ich sie wähle. Da ich aber keine Beziehung zu ihnen aufbauen kann, können sie nie zu meinem „Du” werden (weil sie kein Wort haben). Sie können höchsten zu mir gehören, meinem Ich zugeschrieben werden und somit subjektiv „seinen Wert erhöhen” (der Psychologe

$30 \quad$ Vgl. J. Tischer, Der Streit..., op. cit., S. 248, 277; E. Levinas, Autrement quêtre ou au-delà de l'essence, La Haye 1974, S. 32. 
würde sagen: „das Selbstwertgefühl erhöhen”). Jedoch, obwohl ich in meinem subjektiven Gefühl dadurch „besser” („wertvoller”?) also auch „würdiger, gewählt zu werden” erscheine, verschließe ich mich gleichzeitig gegenüber jeder Möglichkeit, gewählt zu werden, indem ich gegenüber dem Du verschlossen bin. Ich kann mich selbst nicht wählen, so wie ich mich nicht außerhalb von mir stellen kann, ich brauche immer einen „äußeren Stützpunkt”. Ich bin also in einen Teufelskreis geraten: auf der einen Seite wähle ich die „Güter dieser Welt”, um der Wahl des Anderen würdig zu werden, auf der anderen, indem ich mich ihnen zuwende, verschließe ich mich zugleich gegenüber dem Anderen, der mich wählen könnte, und mache es unmöglich, mich als Gutes zu sehen.

Bedenken wir aber auch die Rolle der Aktualität des Wortes. Das Erleben des Du als Gutes (möglich, wie gesagt, durch das Wort) ist nur dann in meinem Leben real (ich wähle ihn als $\mathrm{Du}$ ), und in einem gewissen Sinne „schützt” es den Anderen (Levinas: „du tötest mich nicht”), wenn mir dieses Du in einer aktuell erlebten Beziehung gegeben ist. Es ist also gewissermaßen ein „gegenwärtiges, aktuelles Gutes”, und nicht nur potentiell, gedacht (ideal - im Sinne des monologischen Denkens). Wenn wir diese Beziehung in ihrer Aktualität nicht haben, ist keine Wahl getroffen worden, also ist das Ich nicht „das Gute”. Dann wird auch dieses Ich nicht "geschützt” - mit einem idealen Ich kann ich alles tun, nach Belieben ${ }^{31}$.

Erinnern wir auch noch daran, dass laut Ebner Du - also auch Du als das Gute - mir objektiv im Wort und subjektiv in der Liebe gegeben ist $^{32}$. Eigentlich gibt erst die Liebe dem in mir vorhandenem Wort seine Kraft, im Du das Gute zu sehen, sie stimmt mich also „agathologisch” ein, unterwirft alles der - um mit Tischner zu reden - „Logik des Guten"33. So kann man in der Liebe die Grundlage des Guten sehen.

31 Als Möglichkeit, diesen "Schutz” näher zu erklären, könnte ich mir eine Situation vorstellen, in der jemand in Gedanken auf einen (abwesenden) Anderen wütend schlägt, wenn er jedoch aktuell vor ihm steht, lässt ihn das aktuelle in ihm anwesende Gute nicht wirklich zuschlagen, er wird als ein Du erlebt.

32 Vgl. F. Ebner, Das Wort..., op. cit., S. 25.

33 Vgl. J. Tischner, Der Streit..., op. cit., S. $311 \mathrm{f}$. 
Bonum diffusivum sui - dieses durch Tischner von Meister Eckhart übernommene „Gesetz” der Logik des Guten ${ }^{34}$ spricht aber vom Guten nicht nur als dem Motiv der Wahl, sondern auch von seinem Wachstum und seiner Vermehrung, seiner Verwirklichung. Eine zwischenmenschliche Begegnung, so wie sie in der Perspektive des Ebner'schen Wortes verstanden wird, können wir also auch als die Realisierung, als Verbreitung des Guten ansehen. In dem Verhältnis des Ichs zum Du, das sich im Wort ereignet, wird das Du zum realen Guten für das Ich, und das Ich für das Du. Das Böse hingegen, wenn wir es als das Fehlen oder gar das Gegenteil des Guten sehen wollen, verbindet sich mit der Vereinsamung der Menschen, so wie es das Beispiel Hiobs zeigt ${ }^{35}$. Man kann allgemein sagen, dass sich das Fehlen des Guten mit der Stummheit, mit dem Fehlen des Wortes verbindet. Da wir hier keine genaueren Analysen des Bösen führen können, stellen wir nur fest, dass „das Böse stumm macht”, es versucht, dem Menschen das Wort zu nehmen. Tischner schreibt: „das Böse zeigt sich als eine «Anti-Gravitationskraft», denn es stößt alles und alle $a b^{\text {"36 }}$, es bedeutet „die Unmöglichkeit des Verstehens und Sich-Verständigens auf mehreren Ebenen der Existenz" ${ }^{37}$.

Die Berücksichtigung des Ebner'schen Wortes ermöglicht uns besser den Umstand zu verstehen, dass das Gute sich nur in dem Verhältnis des Ichs zum Du zeigt und wie es in diesem Verhältnis übertragen wird, wie es dort wächst und sich verbreitet. Ohne Wort würde ich im Anderen kein Gutes sehen, ja, ich würde nicht auf die Idee kommen, es zu suchen. Außer der aktuellen Beziehung zum Du wäre ich kein Gutes für den Anderen und gar nicht für mich selbst. Das Wort zeigt uns auch, dass jegliches „einsame Denken” vom Guten abstrahiert und lässt uns es nicht verstehen. Das hat auch Tischner gesehen: „Niemand kann in der Einsamkeit gut sein" 38 - schreibt er, wenn er die Konsequenzen der Dialogphilosophie für seine Agathologie erörtet. Tiefer wird er dieses Thema jedoch bei dem

\footnotetext{
34 Vgl. ebenda.

35 Vgl. ebenda, S. 245f.

36 Ebenda, S. 247.

37 Ebenda, S. 246.

38 Ebenda, S. 359.
} 
Anvertrauen der Hoffnung als Rettung für den Menschen besprechen ${ }^{39}$. Natürlich, die aktuelle Beziehung als eine Verwirklichung des Guten führt uns an das Problem der menschlichen Freiheit bei der Wahl der Beziehung. Dieses Problem wird uns anschließend beschäftigen.

\subsection{Freiheit}

Ich habe bereits angedeutet, dass sich das Problem des Guten mit dem Problem der Freiheit verbindet. Betrachten wir also die Freiheit, indem wir uns an den „Leitseil der Sprache” halten. Die Freiheit bildet natürlich eines der Hauptthemen Tischners, der sie in verschiedenen Kontexten analysiert, vom „ontologischen” angefangen (Freiheit als Kraft, Ursache usw.), über den des Bewusstseins (Freiheit als „Vermögen, einen Neuanfang zu machen”, als „Unvorhersehbarkeit” usw.) bis zum „agathologischen” (Freiheit als Etwas Zwischenmenschliches, bzw. zwischen Mensch und Gott usw. $)^{40}$. Erinnern wir auch noch daran, dass Tischner in seiner Suche die Freiheit immer in Verbindung mit dem Guten versteht („Die Freiheit als Seinsweise des Guten”11), also auch im Dialog - auch wenn ihm ihr Begriff als „sich selbst besitzen” gut gefällt ${ }^{42}$. Ebner widmet dieser Frage nicht so viel Aufmerksamkeit. Dennoch, wenn er den Menschen als denjenigen charakterisiert, der „das Wort hat”, fügt er manchmal hinzu, dass er „im Wort die Freiheit hat" ${ }^{43}$. $\mathrm{Da}$ er dieses Thema nicht weiter bespricht, versuchen wir selbst nach dem Sinn dieser Aussage zu suchen.

Die erste, noch etwas oberflächliche Möglichkeit, wie man diese Aussage verstehen kann, kommt aus der Berücksichtigung der Tatsache, dass bei Ebner das Wort immer mit dem Geist in Verbindung steht, von Balthasars.

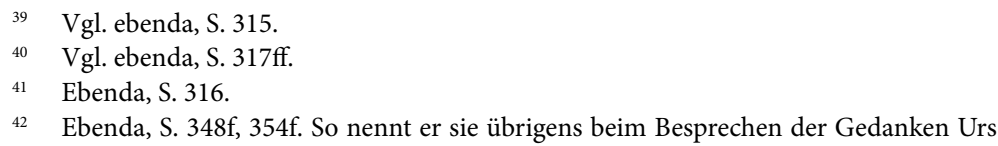

${ }^{43}$ F. Ebner, Zum Wesen der Sprache und des Wortes, [in:] Ders., Schriften, Bd. 1, op. cit., S. 655,660 . 
also auch die Freiheit des Menschen mit seiner Geistigkeit zusammenhängt ${ }^{44}$. Man könnte also meinen, dass es hier einfach um die Unabhängigkeit von der Anschauung geht, was Ebner selbst zu suggerieren scheint. Seine Beschreibung basiert auf dem Unterschied zwischen dem Menschen und dem „geistlosen” Tier. Während im Fall des Tieres (sofern wir das überhaupt bestimmen können), die Anschauung zum Impuls wird, der automatisch (ohne Freiheit) eine instinktive Reaktion auslöst, geht beim Menschen die Anschauung, sobald sie benannt wird, durch das Wort; somit schaltet sich der Geist in den Anschauungsprozess ein und befreit uns von der Unfreiheit der Anschauung. Das, was aus der Anschauung ins Wort verwandelt wird, hört auf, den Menschen zu versklaven. Diese Vision ist jedoch vereinfacht, allein schon deshalb, weil sich das Ebner'sche Verständnis der "geistigen Welt” nie auf ein einsames Ich, sondern auf die „geistigen Realitäten”, d. h. Ich und Du bezieht, also einen dialogischen Charakter hat. Auch die Freiheit muss also dialogisch verstanden werden.

Bevor wir zum Kern unserer Analyse der „Freiheit im Wort” übergehen halten wir zuerst fest, dass, wenn wir ohne tieferes Nachdenken von der Freiheit reden, wir meistens automatisch das gängige Freiheitsverständnis als diese „absolute” Freiheit übernehmen, die uns ohne äußeren Einfluss souveräne Entscheidungen treffen lässt. Dieser Freiheitsbegriff ist jedoch in Frage gestellt worden, allein schon von den Naturwissenschaften. Es ist uns hier nicht möglich, die heutige Diskussion bezüglich Freiheit im Kontext der Gehirnforschung darzustellen, auch wenn diese Problematik einen der zentralen Punkte des aktuellen Streites um den Menschen bildet. Wir beschränken uns auf den Gedanken der Möglichkeit, die „Freiheit des Anderen" zu beeinflussen. Durch das Verabreichen gewisser Medikamente können wir die Stimmung des Patienten so weit verändern, dass er keine Selbstmordgedanken mehr hat, sondern das Leben gern auf sich nimmt - man kann ihn also von den Depressionszuständen weitgehend

44 Die Verbindung zwischen dem Geist und der Freiheit ist natürlich nichts Neues. Hegel schreibt bereits von der Freiheit als dem Wesen des Geistes. Vgl. G. W. F. Hegel, Vorlesungen über die Philosophie der Geschichte, [in:] Ders., Sämtliche Werke, Bd. 5, Stuttgart 1971, S. $22 \mathrm{f}$. 
„befreien”. Wird das Gehirn einem bestimmten elektromagnetischen Feld ausgesetzt, können wir jemanden zu gewissen Handlungen bewegen - hier können wir also seine Entscheidungsfreiheit „einschränken” (oder auch nicht?). In beiden Fällen merkt die betreffende Person meistens nicht, dass ihre Freiheit sich geändert hat. Wenn ich also die Freiheit - auf die vielleicht gängigste Weise - als Fehlen der äußeren Einschränkungen, Bedingungen des einsamen Subjektes in seinen Entscheidungen verstehen will, muss ich zugeben, dass man über eine solche Freiheit gar nicht reden kann, oder dass sie nur eine subjektive Täuschung ist, ein Epiphänomen der Veränderungen im Gehirn.

Im Kontext des Ebner'schen Wortes besteht jedoch ein Unterschied zwischen der Beziehung des Ich zum Du und der Manipulation durch den anderen Menschen. Eine außerbewußte Einflussnahme auf eine andere Person kann man nämlich nicht anders als eine Manipulation nennen, auch wenn sie mit einer guten Absicht geschieht. Merken wir, dass ich beim Manipulieren den Anderen nicht anspreche, sondern über ihn entscheide, ich führe keinen Dialog MIT ihm, sondern einen Monolog ÜBER ihn. Wenn mich also jemand manipuliert, dann haben wir es nicht mit einer Ich-Du, sondern mit einer Ich-Es Beziehung zu tun, mit der Substantialisierung. Gegen diese will ich mich wehren. Und übrigens: Was aber mit der Freiheit des Manipulierenden?

Wenn wir also eine „Freiheit ohne Wort” suchen, eine Freiheit in der Ich-Es Beziehung, verfallen wir erstens in Widersprüche, und zweitens sehen wir, dass hier der „Kampf um die Freiheit” als ein hoffnungsloser Streit einsamer und de facto unfreier "Ichs” vor sich geht, jeder gegen jeden.

Vielleicht können wir jedoch diese Vision verlassen und woanders nach der Freiheit suchen: erstens nicht in der Einsamkeit (Ich-Es) sondern in einer Beziehung (Ich-Du), zweitens nicht im Konflikt mit dem Anderen, sondern in einer "Zusammenarbeit”. Diesem Prinzip folgend muss ich folgendermaßen denken: der Andere ist für mich weder Beschränkung noch Bedrohung der Freiheit, sondern eine Chance, sie zu erreichen - durch das „rechte Wort”. Wir können es durch ein Bild veranschaulichen: wenn ich mich in einem isolierten Raum vor dem 
Anderen verschließe, weil ich meine Freiheit verteidigen will, werde ich gerade dadurch „eingesperrt", also der Freiheit beraubt. Wenn ich aber ein Wort an Du richte, befreie ich Dich zu einer Beziehung mit meinem Ich. Man kann auch mit Tischner sagen, ich "schaffe” oder "gebäre” Dich, ich schaffe in dir ein Du. Nicht „Sich-Verschließen” lässt mich also meine Freiheit bewahren oder erst erreichen, sondern die Suche nach dem „rechten Wort”. Es geht dabei darum, mich von der Verschlossenheit hinter meiner "chinesischen Mauer” zu befreien. Dies geschieht nicht anders als durch das rechte Wort. In diesem Fall "schaltet sich der Geist in den Anschauungsprozess" auf eine andere Weise ein: eine Anschauung führt mich zu dem Verhältnis zum Du und dadurch zur Freiheit.

Wenn wir die Freiheit dialogisch verstehen, müssen wir auch merken, dass ich mit jemand wesentlich zur Freiheit Unfähigem, also auch mit einem unfreien Ich (wenn es ein solches überhaupt gibt, man müsste hier eher von einem „Nicht-Ich” reden) keine Beziehung haben kann. Erinnern wir hier an die polnische Dichterin und Nobelpreisträgerin Wisława Szymborska und ihr „Gespräch mit dem Stein, wo alle Versuche scheitern, den Stein zum Du zu machen. Der Stein, wie die Lyrikerin schließlich sagt, „hat keine Tür” um Zutritt zu gewähren. Szymborska lässt den Stein antworten: „Du kannst mich kennenlernen, du wirst mich aber niemals erkennen" ${ }^{45}$, als ob sie den wesentlichen Unterschied zwischen einer Ich-Du und einer Ich-Es Beziehung suggerieren möchte. Der Stein „hat kein Wort”, ich kann ihn also nicht ansprechen, ich kann nicht „zu ihm” sondern höchstens „über ihn” reden.

Die Dinge sehen anders aus, wenn wir uns eine Welt der Sklaverei vorstellen, und dass wir etwas von einem „fremden” Sklaven brauchen. Auch hier muss ich nicht den Sklaven ansprechen, nicht er ist mein Gesprächspartner, ich rede nicht „zu ihm”, sondern „über ihn” zu dem, der über ihn entscheidet, der das wirkliche Subjekt ist, der frei

45 W. Szymborska, Sto wierszy - Sto pociech, Hundert Gedichte - Hundert Freuden, Ausgewählt und übertragen von Karl Dedecius, Kraków 1997, S. 250 f. 
ist - zu seinem Herrn. Erst die Freiheit bewirkt also, dass ich es mit einem echten Ich zu tun habe. Wir könnten auch sagen, dass der Sklave - in einem ganz konkreten Sinne - „sich nicht besitzt”. Wenn wir es aber tiefer betrachten, müssen wir merken, dass auch der Sklave ein Mensch ist, also „das Wort hat”, und so kann ich ihn doch ansprechen. Mehr noch, mein Ansprechen kann in ihm ein Du „befreien”, oder wie Tischner sagt, „gebären”. Dieses „Entstehen” des Du im Sklaven ist zugleich das Entstehen seiner Freiheit. Und dank dieser zuerst „inneren” Freiheit wird der Mangel an äußerer Freiheit sichtbar. Das führt uns zu der wichtigen Entdeckung, dass indem ich an jemanden das Wort richte, ich ihn zugleich befreie. Erst nach dem Ansprechen - also im Dialog - können wir von der Freiheit reden. Die Freiheit ist also tatsächlich ursprünglich im Wort.

Auf der anderen Seite könnten wir mit Tischner sagen, dass erst wenn ich „mich selbst besitze”, eröffnet sich vor mir die Möglichkeit, mich als Gutes zu sehen, und die Möglichkeit, mich als Gabe zu schenken, also auch die Fähigkeit zu einer Beziehung. Die Freiheit als „sich selbst Besitzen” entspricht der Umwandlung ins Wort, denn „besitzen”, oder "haben” kann ich nur, was ich ins Wort fassen kann. So kann man sehen, dass es schwer möglich ist, von diesem „sich selbst besitzen” in der Einsamkeit zu sprechen, allein schon deshalb, weil mich die Sprache zwangsläufig zum Du führt. Auch Tischner sagt ja: „Niemand kann in der Einsamkeit gut sein. Und ferner kann niemand aus Notwendigkeit gut sein" ${ }^{\text {. }}$. Und auch: „Ich bin frei, wenn ich dem Anderen die Freiheit zugestehe" ${ }^{\prime 7}$. Vielleicht sollen wir also, wenn wir dialogisch denken, nicht von „besitzen oder besessen sein” reden, sondern eben von der Offenheit auf das Wort hin?

Wenn wir an die Aktualität des Wortes denken, betonen wir, dass auch die Freiheit eher dynamisch gesehen werden soll, als aktuelles Befreien durch das Wort und nicht als eine „bestehende”, gar statische Eigenschaft der Person. Ebner beruft sich auf eine Situation in der

$46 \quad$ J. Tischner, Der Streit..., op. cit., S. 359.

47 Ebenda, S. 350. 
zwischenmenschlichen Begegnung, wo das Aussprechen des „rechten Wortes" ein Gefühl der Entlastung, Befreiung, sogar der Erlösung bewirkt $^{48}$. Wir lesen dort:

Es gibt nichts wahrhaft Menschliches, dem nicht das Wort entspräche, nichts Menschliches, das in seiner Dunkelheit und Unerklärlichkeit, in seiner inneren Gebundenheit und Verborgenheit im rechten Wort nicht seine Klärung und Erklärung, seine Befreiung und Offenbarung erführe ${ }^{49}$.

Wir können aber auch fragen, wovon befreit uns das „rechte Wort”? Zweifellos von der inneren Verzwickung, Unklarheit, Verschlossenheit, Einsamkeit, Lüge. Es geht dabei um eine Befreiung des Menschen von seinem Nicht-Ich zum wahren, freien Ich, darum also, es mit Freiheit zu versehen ${ }^{50}$. Hier könnten wir kurz das Feld der rein philosophischen Analysen verlassen und bemerken, dass das Konzept der „Freiheit im Wort" in Übereinstimmung mit der Psychoanalyse stehen würde (wo die Benennung des Problems der Anfang der Befreiung ist) ${ }^{51}$ aber auch mit der Bibel, besonders mit den Johanneischen Schriften.

Betrachten wir unser Thema auch noch von der Seite der alten Überzeugung von der Verbindung zwischen dem Geist und der Freiheit: wenn ich in Dir den Geist erwecken will (den Geist im Ebner'schen, also dialogischen Sinne), kann ich das nur (objektiv gesehen) im Wort, das (subjektiv) von der Liebe begleitet wird, in dem "rechten Wort”. Gleichzeitig erwecke ich die Freiheit in mir selbst, oder werde vielleicht durch die Antwort des Du befreit. Ich werde befreit zu einer Beziehung, als Resultat der Intervention des Wortes in den Anschauungsprozess.

48 Vgl. F. Ebner, Zum Problem der Sprache und des Wortes, [in:] Ders., Schriften, Bd. 1, op. cit., S. 680 .

49 Ebenda.

${ }^{50}$ Mit Tischner schauen wir die Gestalten aus der „Trauung” des polnischen Schriftstellers Witold Gombrowicz: ob das Ansprechen einer solchen Person mit dem „rechten Wort” nicht aus ihrem „Nicht-Sein” erfolgt, mit dem sie nicht zurechtkommt?

${ }^{51}$ Ebner studierte auch die Schriften von Freud (dem er jedoch Mangel an Respekt gegenüber dem Menschen, seine Substantialisierung vorwirft), er schätzte aber vor allem die Individualpsychologie Alfred Adlers. 
Interessant ist, dass in jedem dieser Fälle die Freiheit ursprünglich „von Außen kommt", ich kann sie selber nicht erwirken, indem ich mich in meinem Ich einschließe.

Wir kommen hier aber zu einem Paradoxon, typisch für die Freiheitsanalysen: damit die Freiheit kommt, muss ich sie empfangen, ich muss mich „befreien lassen” - also brauche ich letzten Endes zumindest eine Anlage zur Freiheit. In diesem Kontext stellte sich Tischner die Frage, was bewirkt im Menschen diese rätselhafte Umwandlung, diesen "unmöglichen Sprung von Verzweiflung zu Hoffnung"52 (in unseren Worten: von der Verschlossenheit zur Offenheit, zur Liebe)? Alle Versuche einer reduktionistischen Erklärung einer solchen Freiheit, aus dem Gesichtspunkt der 3. Person (z. B. eine Dosis Medikamente) sind nicht zufriedenstellend, denn sogar nach den Medikamenten verhalten sich Personen unterschiedlich. Wir haben es hier offensichtlich mit einem Geheimnis des Menschen par excellence zu tun.

In diesem Punkt eröffnet sich aber auch der Weg zum Konzept der Gnade und der Suche nach der göttlichen Abstammung der Freiheit ${ }^{53}$. Der Eindruck besteht aber, dass auch hier das Wort eine entscheidende Rolle spielt, das Wort in ihrer "göttlichen” Dimension.

Weisen wir noch darauf hin, dass das Wort sich ja mit dem Guten verbindet und seine Logik teilt. Das Gute wird also im Wort realisiert, so, dass das Ich durch das in der Aktualität seines Gesprochenwerdens gefasste Wort das Du befreit, es als Gutes sieht und zum Guten macht, und wird von ihm selbst befreit. Das Gute befreit das Gute, die Freiheit setzt Freiheit frei. „Niemand kann in der Einsamkeit gut sein. Und ferner kann niemand aus Notwendigkeit gut sein"54 - diese Aussage Tischners scheint hier eine neue Bedeutung anzunehmen. „Ohne Freiheit kein Gutes”, auch in dem Sinne: „Wenn ich nicht frei bin, wenn ich eingeschlossen hinter meiner chinesischen Mauer bin, kann ich nicht gut sein und nichts Gutes bewirken". 


\subsection{Hoffnung}

In seinem Artikel über das Verständnis der Hoffnung bei Tischner sieht Jarosław Jagiełło die Hoffnung zuerst als einen gewissen „Horizont der Erkenntnis"55, die Hoffnung wird also im Kontext der Erkenntnis gesetzt. Die Rolle des Horizonts ist es, durch eine gewisse „Anziehung” die Erkenntnis zu ermöglichen. Die Hoffnung wäre also das, was zur Erkenntnis „anzieht”. Tut sie das auch zur Beziehung des Ichs zum Du?

Wenn wir von der Erkenntnis im Kontext der Beziehung des Ichs zum $\mathrm{Du}$ sprechen, müssen wir immer auf die Falle des intentionalen Subjekt-Objekt-Denkens achten, das von beiden unseren Denkern eindeutig verworfen wird. Es geht uns also hier nicht um eine Erkenntnis im Sinne der episteme, um den Versuch einer „wissenschaftlichen” und auch nicht einer ,objektiven” Erkenntnis - welches laut Ebner nur einen abstrahierten Teil des Ich, ein gewisses „objektives Ich”, aber eigentlich nur ein zur Unkenntnis verstelltes reales Ich betrifft. Tischner beschreibt ein solches Ich als den mythischen Gyges „der alles sieht und selbst unsichtbar bleibt"'56. Diese Erkenntnis würde laut Levinas das Gebiet des Bedürfnisses, und laut Tischner nur die „Bühne des Dramas” betreffen. Mit einer solchen Erkenntnis verbindet sich auch die Problematik der Macht und der Herrschaft über den Anderen, aber nicht der Beziehung des Ichs zum Du. Einen realen Menschen wird man so nicht „erkennen” können.

Wenn wir also die Falle des intentionalen Denkens in unseren Analysen der Hoffnung umgehen, müssen wir zugeben, dass es auch hier die Hoffung ist, die mich zum Eintritt in die Beziehung zum Du „anzieht”, die Hoffnung auf ein Gutes. Dieses Gute - wie bereits beschrieben - ist mit Wort verbunden.

Die Hoffnung ist also eine Erwartung des Guten. Auf diese Weise wird uns die zeitliche Dimension der Hoffnung sichtbar. Die Hoffnung bezieht

55 Vgl. J. Jagiełło, Problematyka nadziei we współczesnej filozofii człowieka, [in:] http:// www.kst.kielce.opoka.org.pl/Jagiello.htm (1.07.2012). Der Artikel ist auch erschienen in: „Kieleckie Studia Teologiczne", 2004, nr 2, S. 49-69.

56 J. Tischner, Między pytaniem a odpowiedzia, czyli u źródeł obiektywizmu, „Studia Filozoficzne”, 1983, nr 5/6, S. 115-125; Ders., Der Streit..., op. cit., S. 279. 
sich nämlich immer auf die Zukunft, aber ihre Wirkung geschieht in der Gegenwart. Möglich ist sie allerdings dank der Vergangenheit. Um ein Gutes zu erwarten, muss ich es bereits irgendwie kennen, es erfahren haben. Wie kann ich es aber kennen, wenn nicht aus der Beziehung zum Du, in der es sich mir mitteilt, wobei mir diese Beziehung im Wort zugänglich ist? Jemand hat mich gewählt, also als Gutes erkannt. Auch ich habe ihn als Gutes erkannt - und mich vielleicht noch stärker als Gutes gefühlt (nach dem Prinzip bonum diffusivum sui). So werde ich es auch künftig in der Beziehung zum Du suchen, im Wort. Die Grundbedingung der Hoffnung ist also das Wort und die in ihm zugängliche Beziehung zum Du.

Es gibt also keine Hoffnung ohne Du, keine Hoffnung ohne Wort! So wie es auch kein Ich und kein Gutes - sowie keine Freiheit ohne Wort gibt. Damit ich ein Gutes bin, muss mich jemand zu seinem Du wählen, an mich sein Wort richten. Die "Güter dieser Welt" sind dabei keine Alternative. Auch eine „Entzweiung des Selbst”, wo das eine Ich das andere wählen könnte, reicht hier nicht. Es muss jemand sein, der „mir seine Hoffnung anvertraut”, ein Gutes von mir erwartet. Kann ich mir selbst meine Hoffnung anvertrauen? Welches Gute würde ich davon erwarten, wenn das Gute erst in der Beziehung zum realen Du möglich ist? Trügerisch würde diese Hoffnung sein, die auf einem „entzweiten Selbst" oder fiktiven Du beruht.

Ich habe oben gesagt, dass das Gute vor allem das Du selbst, das Ich selbst ist. Denken wir aber daran, dass das Ich in der Begegnung mit dem $\mathrm{Du}$ "geboren” wird, wenn die Liebe das Wort begleitet. Die Hoffnung ist also in erster Linie eine Erwartung auf die „Realisierung” des Ich - und zugleich des Du, als der geistigen Realitäten, und sie werden in einer Beziehung des Ich zum Du, zum realen, aktuellen Du „realisiert”. Es ist kein Zufall, dass Ich erst in der Beziehung zum Du mich als eine „Realität” empfinde. Sonst könnte ich mit den - von Tischner zitierten - Gestalten aus der Trauung von Gombrowicz sagen, „ich war es nicht, mich hat es nie gegeben", und dass ich höchstens meine Menschlichkeit „rezitiert habe”57. In Wirklichkeit kann ich erst dann mein Ich 
entdecken, bzw. „mich entfalten, aufblühen sehen”. Das würde wieder der Logik des Guten entsprechen, in der das Gute Gutes gebiert.

Im Laufe unserer Überlegungen sind wir also zum für Tischner eigenen „Anvertrauen der Hoffnung” übergegangen ${ }^{58}$. Ich vertraue meine Hoffnung dem an, der mir ein Versprechen gibt ${ }^{59}$. Da merken wir noch einmal, dass das „Anvertrauen der Hoffnung” erst durch Wort und im Wort möglich ist. Die Hoffnung stützt sich aber nicht nur auf das Wort (Verhältnis des Ichs zum Du), sondern beruht auch auf den „Worten”, auf dem, was in diesem Verhältnis gesagt wird. Diese „Worte” können aber auch zu „leeren Wörtern” werden, ohne Bezug auf die konkreten geistigen Realitäten (Ich und $\mathrm{Du}$ ) - so wie bereits erwähnte Gestalten aus der Trauung von Gombrowicz - also Wörter, die nicht von einem realen Du kommen, sondern von irgendeinem Wortspiel ${ }^{60}$. Auf solche Worte kann sich die Hoffnung nicht stützen. Die Hoffnung entsteht in einer konkreten, aktuellen Sprachsituation (also in der Beziehung), aus dem Hören auf das „rechte Wort”, und nicht beim Lesen der Romane oder Studieren der mathematischen Formel. Sie bezieht sich auf mich als ein konkretes Ich, oder als Du eines konkreten Ich. Die Hoffnung ist also auch kein "gewöhnliches", sondern ein gewissermaßen „garantiertes" Erwarten des Guten, garantiert eben im Wort, ähnlich wie im Wort, im „rechten Wort” ist mir das Du garantiert. Meine Hoffnung kann ich nur einem realen, konkreten, im rechten Wort gegebenen Du anvertrauen.

Die Position des Wortes in der Hoffnung erfassen wir auch in dem tiefen - seine wahre Tiefe wird erst im Kontext des Ebner'schen Wortes sichtbar - Ausdruck „das Wort geben” (z. B. „ich gebe dir mein Wort”). Indem ich „das Wort gebe”, erwecke ich die Hoffnung, und zugleich "gebäre" ich das Du als eine geistige Realität im anderen Menschen. Dies sagt uns sehr viel von dem Wesen der Ich-Du-Beziehung. Wenn ich „das Wort gebe”, gebe ich zweifellos auch mich selber mit, natürlich

58 Vgl. J. Jagiełło, Problematyka..., op. cit.; J. Tischner, Der Streit..., op. cit., S. 315.

59 Vgl. J. Tischner, J. Żakowski, Tischner czyta Katechizm, Kraków 1997, S. 92. Vgl. J. Jagiełło, Problematyka..., op. cit.

${ }^{60}$ Vgl. J. Tischner, Der Streit..., op. cit., S. $92 \mathrm{f}$. 
ohne Garantie, dass ich es „wieder bekomme”, oder etwas dafür erhalte. Vielleicht drückt sich so dieser Heroismus des "auf sich nehmen” der Hoffnung des Anderen aus, von dem Tischner in der Welt der menschlichen Hoffnung spricht ${ }^{61}$ ?

Den Ausdruck „das Wort geben” könnten wir auch in „das Wort verleihen” umformulieren, denn die eigene „Logik des Wortes” bewirkt, dass ich mein Wort, wenn ich es dem Anderen schenke, nicht verliere, sondern es "redupliziere”, verdopple, so wie sich das Licht der Kerze durch das Anzünden einer anderen verdoppelt. Ich kann aber natürlich nur dem ein Wort geben, der bereits als Mensch „das Wort hat" und mein Du werden kann.

Es funktioniert natürlich im Deutschen genauso der Ausdruck ,das Wort verlieren”. Das ist gewissermaßen auch möglich - obwohl nie in einem vollen Sinn - wenn ich nach gewissen Enttäuschungen, Misserfolgen, „Leiden am Nächsten” die Liebe verliere, also die Offenheit, wohl auch den "Glauben” an den Anderen. In dieser Lage, auch wenn ich immer noch als Mensch „das Wort habe”, lasse ich mich vom Wort des Anderen nicht erreichen und richte selbst kein Wort an ihn. Tischner bezeichnet den Menschen in dieser Lage als eine „Monade mit zugeschlagenen Fenstern”62. Von dem „Verlieren der Worte” redet man am häufigsten vielleicht in der Form „keine Worte drüber verlieren” und im Kontext ihres ziellosen Aussprechen, wenn ich annehme, dass sie sowieso nicht vom Anderen angenommen werden. Wer auf diese Weise „das Wort verloren" hat, wird wahrscheinlich nicht so schnell „das Wort geben", hat also tatsächlich - wiederholen wir aber: nur in einem gewissen Sinne - das Wort verloren, ist „stumm” geworden. Man braucht nicht hinzufügen, dass er damit auch die Hoffnung verloren hat.

Die Hoffnung wird also als die Erwartung eines Guten gesehen, aber als eine „im Wort, durch ein reales Du garantierte” Erwartung. Diese Erwartung geschieht in der Zeit, die Hoffnung betrifft nämlich die $\mathrm{Zu}-$ kunft, passiert aber in der Gegenwart, genauso wie die Beziehung zum

${ }_{61}$ Vgl. Ders., Świat ludzkiej nadziei, op. cit., S. 98, $305 \mathrm{ff}$.

62 Ders., Der Streit..., op. cit., S. 267 f. 
konkreten $\mathrm{Du}$, auf der sie aufbaut. Das Gute, das von der Hoffnung erwartet wird, kann nicht von einer „zeitlosen” Idee kommen, welche in meinem Verstand geboren oder - um mit Ebner zu sprechen - vom „Leben der Generation” übernommen wird. Sie kann auch nicht in der Einsamkeit entstehen. Das erwartete Gute kommt von Außen und wird von Außen garantiert, vom Anderen oder von "Außerhalb der Monade” (wie sich vielleicht Tischner ausdrücken würde), oder von einem realen, konkreten Du (Ebner $)^{63}$. Eine Hoffnung in der Einsamkeit wäre lediglich eine Flucht des Menschen in die Zukunft: auf später verschieben, oder gar auf ein Leben im Jenseits, ein „ewiges Leben”. Ebner zitiert in diesem Kontext die Worte Pascals von dem, der „nicht wirklich lebt, sondern nur erwartet zu leben - und darüber vergeht die Zeit"64. Wenn die Hoffnung eine solche Flucht wäre, dann wäre sie auch eine Absage an die Gegenwart, also auch eine Absage an das Gute, an sich selbst.

\section{Der Mensch des Wortes}

Indem wir versuchten, uns konsequent an den Ebner'schen Leitseil des Wortes zu halten, haben wir also drei „Worte” der Tischner'schen Philosophie und zugleich drei Grundmomente des menschlichen Lebens betrachtet. Dies hat es uns ermöglicht, ihren Platz wesentlich - um mit Tischner zu sprechen - „im Horizont der zwischenmenschlichen Begegnung” zu sehen. So erscheint die Rolle des Wortes im Verstehen der Freiheit, der Hoffnung und des Guten als unersetzlich. Bedeutet dies, dass sich in der Tiefe der Anthropologie Tischners das Ebner'sche Wort versteckt?

Ich habe einen folgenden Eindruck: Wie aus der Lektüre des „Menschlichen Dramas” entnommen werden kann, ist es Tischner versagt geblieben, das Ebner'sche Konzept des Wortes gründlich zu erforschen, obwohl er angeblich eine solche Absicht hatte. Seine Sympathie zur Dialogphilosophie verdankt er eher Levinas, der zusammen mit Rosenzweig meint,

63 Vgl. F. Ebner, Das Wort..., op. cit., S. 35ff.

64 Ders., Wort und Liebe, op. cit., S. 912. 
dass die dialogische Beziehung den Menschen direkt engagiert, ohne irgendein Buber'sches „Zwischen” (ein Resultat des Nachdenkens über das Ebner'sche Wor ${ }^{65}$ ?). Tischner beruft sich also eher auf Levinas (der auf die jüdischen Quellen zugreift, und für den das Hauptprinzip das im Herzen verspürte Gesetz ist ${ }^{66}$ ) - auch wenn er diesen Gedanken aus dem christlichen Standpunkt kritisiert, erweitert und weiterentwickelt. Daher kann er auch nicht gleichzeitig den durch das Johannesevangelium inspirierten Ebner mit seinem Wort berücksichtigen, dem Wort verstanden als Sprache und Beziehung. Es kommt mir also vor, dass unser „Denken am Leitseil der Wortes" uns in der Anthropologie Tischners bisher unentdeckte Aspekte sehen lässt und dass sein ganzes Konzept uns wenn nicht deutlicher, dann zumindest im neuen Licht erscheint. Der Mensch der Hoffnung, der Freiheit und des Guten erweist sich in seiner Tiefe als der Mensch des Wortes.

65 Nach den Schriften von Rivka Horwitz ist es allgemein bekannt, dass Buber noch vor der Formulierung seiner Hauptthesen des Buches „Ich und Du” die Werke Ebners gelesen hat und sein Denken kannte. Vgl. R. Horwitz, Ebner und Buber, Rosenweig und Ehrenberg, [in:] Gegen den Traum vom Geist, Beiträge zum Symposion von Gablitz 1981, Hg. W. Methlagl et al., Salzburg 1985, S. 97-105. Vgl. M. Buber, Nachwort. Zur Geschichte des dialogischen Prinzips, [in:] Ders., Das dialogische Denken, Heidelberg 1973, S. 309.

${ }^{66}$ Adam Hernas schreibt in Bezug auf das Substitutionsprinzip Levinas': „Sein Prinzip ist hier das höchste Gesetz, dessen Quellen in vorhistorischen und außerzeitlichen Akt der Schöpfung zu suchen ist, ein Gesetz, das über die Schuld entscheidet und dem Gericht des Anderen unterstellt". Vgl. A. Hernas, Tischner i Levinas. Księdza z rabinem spór o człowieka, [in:] http://www. tischner.org.pl/artykulym.php?aid=45, übers. von K. Skorulski (1.07.2012). 